\title{
Cyclic Single Atom Vertical Manipulation on a Nonmetallic Surface
}

\author{
David Abbasi-Pérez,* Hongqian Sang, Filipe L. Q. Junqueira, Adam Sweetman, J. Manuel Recio, \\ Philip Moriarty, and Lev Kantorovich*
}

Cite This: J. Phys. Chem. Lett. 2021, 12, 11383-11390

Read Online

ABSTRACT: Motivated by the quest for experimental procedures capable of controlled manipulation of single atoms on surfaces, we set up a computational strategy that explores the cyclical vertical manipulation of a broad set of single atoms on the GaAs(110) surface. Firstprinciples simulations of atomic force microscope tip-sample interactions were performed considering families of GaAs and Au-terminated tip apexes with varying crystalline termination. We identified a subset of tips capable of both picking up and depositing an adatom ( $\mathrm{Ga}, \mathrm{As}, \mathrm{Al}$, and $\mathrm{Au}$ ) any number of times via a modify-restore cycle that "resets" the apex of the scanning probe to its original structure at the end of each cycle. Manipulation becomes successful within a certain window of lateral and vertical tip distances that are observed to be different for extracting and depositing each atom. A practical experimental protocol of special utility for potential cyclical manipulation of single atoms on a nonmetallic surface is proposed.

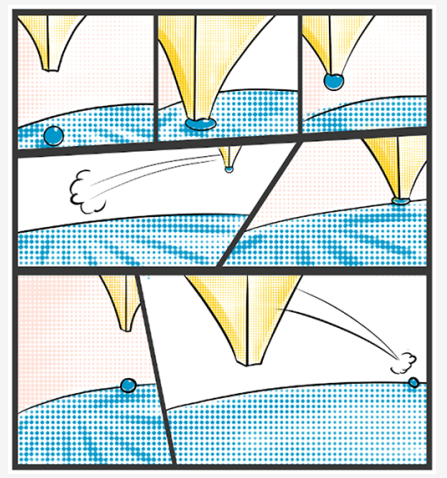

ince the invention of scanning probe microscopes in the $\checkmark 1980 s^{1-3}$ there has been a series of astounding advances made in our ability to image, probe, and manipulate matter at the single atom - and, more recently, single chemical bondlimit. Individual atoms and molecules have been pushed, pulled, poked, prodded, or otherwise picked-and-placed by using a variety of manipulation schemes underpinned by a range of physicochemical interactions. ${ }^{4}$ Building on the substantial achievements of scanning tunneling microscopy (STM) in atomic and molecular manipulation, ${ }^{5-12}$ dynamic force microscopy, with both conventional silicon cantilevers ${ }^{13-15}$ and the qPlus sensor design introduced by Giessibl, ${ }^{16-19}$ has led to a step change in precision and, equally importantly, substantially extended the palette of manipulation mechanisms available to probe for microscopists. These now include, in particular, the controlled application of mechanochemical force. ${ }^{11,12,20}$ This type of mechanochemistry strategy reaches its apogee in the direct, bond-by-bond, and chemical synthesis of single molecules, as described in, for example, the pioneering papers of Pavilcek et al. ${ }^{21,22}$

The effective rate-limiting component in any probe microscopy technique, however, is of course the probe itself; coercing the apex of the tip into the precise atomic or molecular structure required to image or modify matter at the atomic level is a major bottleneck when it comes to increasing the efficiency of the technique. Gross et al.'s ${ }^{23}$ demonstration that tip functionalization via the attachment of a $\mathrm{CO}$ molecule could dramatically improve image resolution for force microscopy has led to the widespread adoption of that protocol, particularly in the qPlus variant. ${ }^{19}$ Strategies for tip functionalization other than $\mathrm{CO}$ attachment have also been used very successfully. ${ }^{24-26}$ It is safe to say, however, that control of the tip apex remains an especially challenging aspect of scanning probe microscopy. Machine-learning approaches have exciting potential when it comes to automating tip control, ${ }^{27-29}$ but the development of strategies to optimize the probe apex requires a more considered combination of experimental and theoretical input. In particular, the atomic, chemical, and electronic structure of the tip apex not only establish image quality but also fundamentally underpin our ability to manipulate atoms and molecules. For example, relatively small changes in the orientation of a tip-terminating atomic orbital can have significant ramifications on single atom manipulation. $^{30}$

Pushing the limits of single atom control beyond the creation of $2 \mathrm{D}$ structures and toward the fabrication of more complex nanostructures necessitates a careful consideration, and systematic sifting, of the very wide structural space associated with the large family of potential tip states. At a minimum, it requires that the tip be able to pick up an atom at one position on the surface and subsequently deposit it elsewhere, that is, to carry out so-called "vertical" manipulation of atoms in a routine and reliable manner, with atomic resolution retained throughout the entire process. Sugimoto and co-workers ${ }^{31}$ introduced a key mechanism for manipulation of this type: the "vertical interchange" protocol.

Received: July 14, 2021

Accepted: August 3, 2021

Published: November 16, 2021 

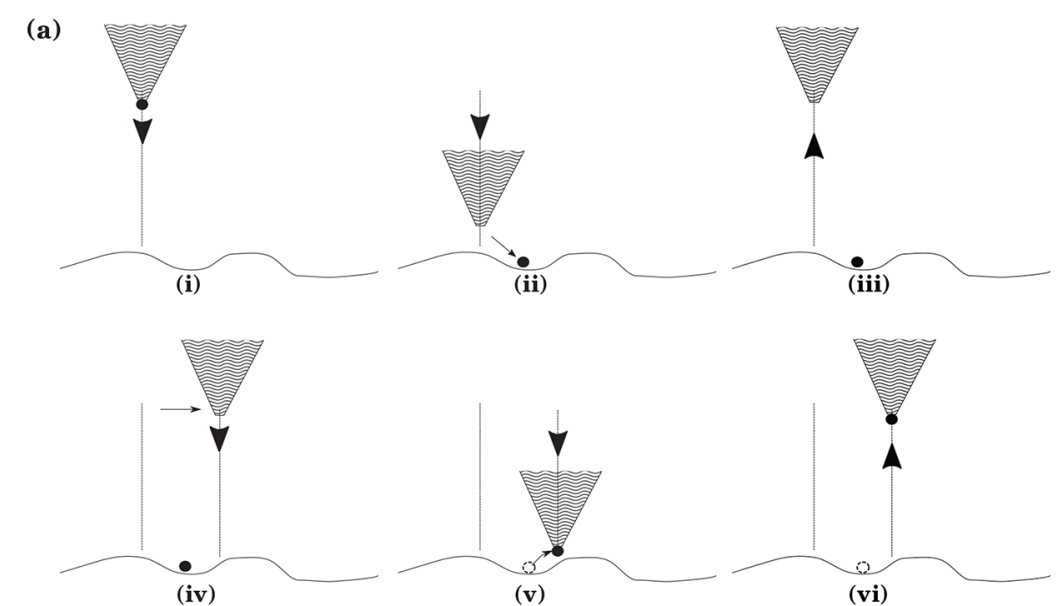

(b)
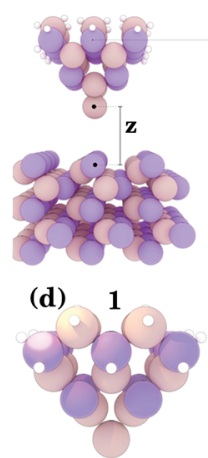

(c)
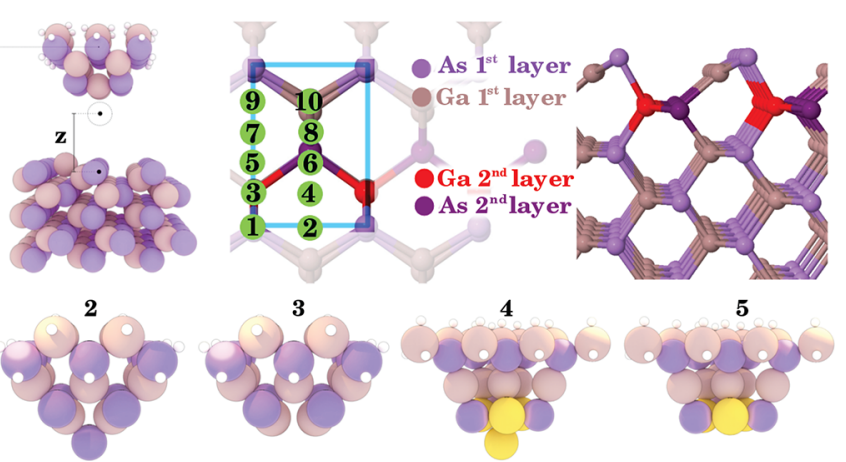

Figure 1. (a) Schematics of the full single atom deposition-picking cycle. The original atomically sharp tip in (i) is recovered in (vi). (b) Definition of the tip-surface distance $z$ used in our simulations. (c) Optimized GaAs(110) surface: top view (left) with the lateral grid points indicated in green and side view (right). (d) GaAs tips based on the GaAs(001) surface with Ga (tip 1) and As (tip 2) atoms at their apexes and the common blunt tip 3 without those apex atoms. Doped GaAsAu tips based on the GaAs(111) surface, with (tip 4) and without (tip 5) the Au apex atom. Pale pink, purple, yellow, and white atoms are gallium, arsenic, gold, and hydrogen, respectively.

Subsequently, a number of other vertical manipulation strategies have been reported. ${ }^{12,26,32,33}$ However, using the same tip to manipulate different types of atoms on a semiconducting surface repeatedly during the same experimental run (and hence with the same tip) still remains elusive. The atomic manipulation process described below represents a new and important addition to the class of vertical manipulation methods, with an ability to manipulate a wide class of different atoms individually with the same tip within the same experimental run.

An obvious advantage of the vertical manipulation protocol is that it does not require any tunnel current or application of a voltage pulse (and therefore works on insulating surfaces) and that because it is a vertical exchange mechanism the material can be moved from anywhere on the surface unlike in lateral manipulation modes.

In searching for tip types that are capable of cyclical (i.e., structurally reversible) manipulation, we focused here, using exclusively a theoretical approach based on $a b$ initio density functional theory (DFT) calculations, on broad structural features, dynamics, and properties rather than on specific atomistic detail as the latter is difficult, if not impossible, to replicate in experiment. Remarkably, our DFT calculations have identified a class of tips that not only enable cyclical manipulation but also are chemically nonspecific: with these tips a variety of atoms, including $\mathrm{Au}, \mathrm{Ga}, \mathrm{As}$, and $\mathrm{Al}$, can be picked up and dropped, with atomic precision, returning the tip apex to its original structure in each case. We predict an energy-distance variation for this tip type that can be used in experiments as a characteristic signature of its presence. And, most importantly, on the basis of our theoretical predictions, we discuss possible ways in an experiment of fabricating such types of "self-restoring" tips and performing the manipulation experiments with them.

The entire remove-deposit cycle can be divided into a number of elementary movements of the tip, as summarized in Figure 1a: (i) An atomically sharp tip is located above a specific lateral position on the surface. (ii) The tip is driven into the surface within a specific window $\Delta z$ of tip heights, and the apex atom jumps from the tip to the surface, becoming a surface adatom while the tip becomes blunt. (iii) Then the tip is retracted keeping the same lateral position; we have obtained a reactive blunt tip that can perform manipulation events from now on. (iv) The blunt tip is moved to a different lateral position on the surface next to a surface adatom we intend to pick up. (v) Another approach is performed at a certain lateral position, and at a very close tip-surface distance the atom jumps from the surface to the tip. (vi) The tip is retracted again, recovering the original structure. The picked atom can now be deposited somewhere else on the surface (steps i-iii). This way the full vertical manipulation cycle is performed.

In practice, in each approach-retraction process the tip is positioned in a certain way with respect to either the atom to be picked up or the lattice site where the apex atom is to be dropped. Approach-retraction simulations were performed for many lateral tip positions and for a range of approaching 


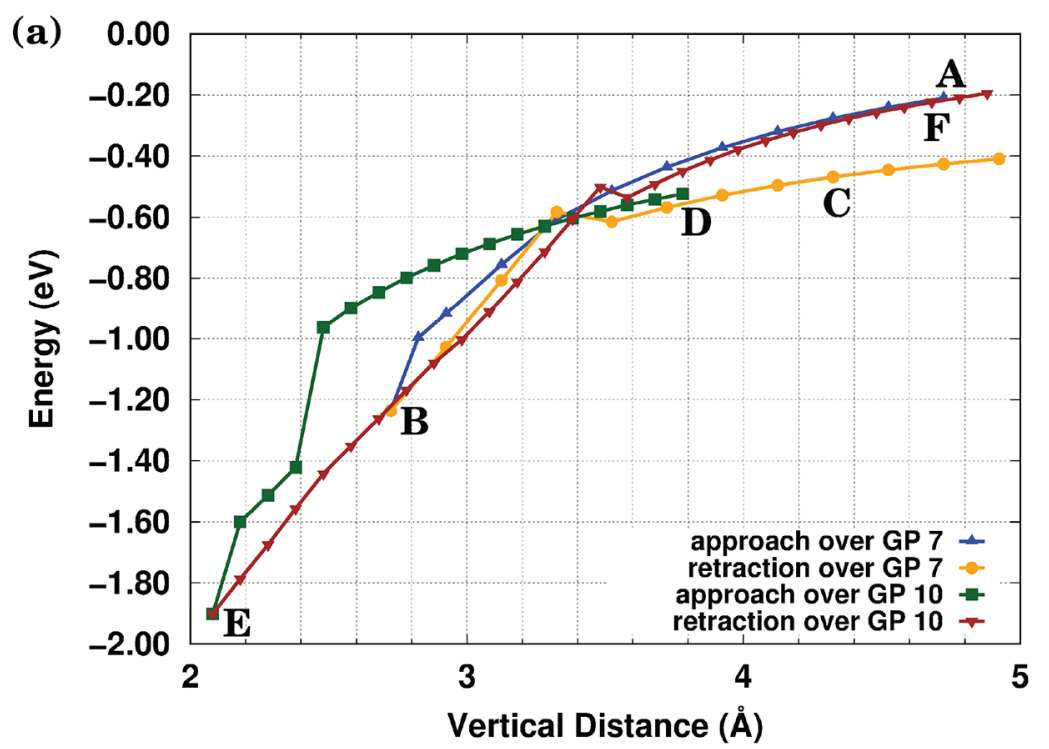

A

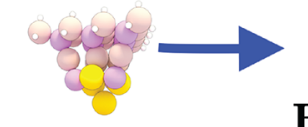

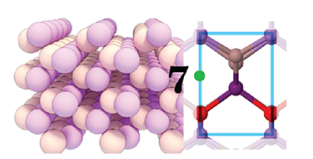

B

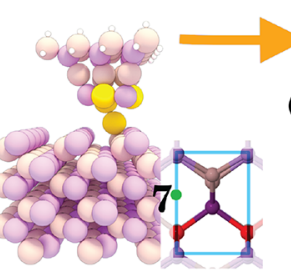

C
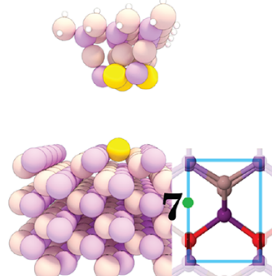

D

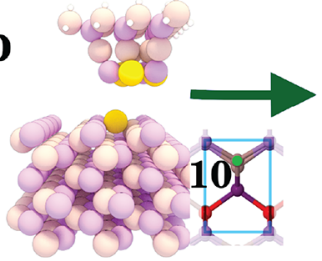

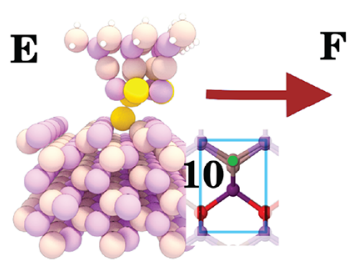

\section{.}



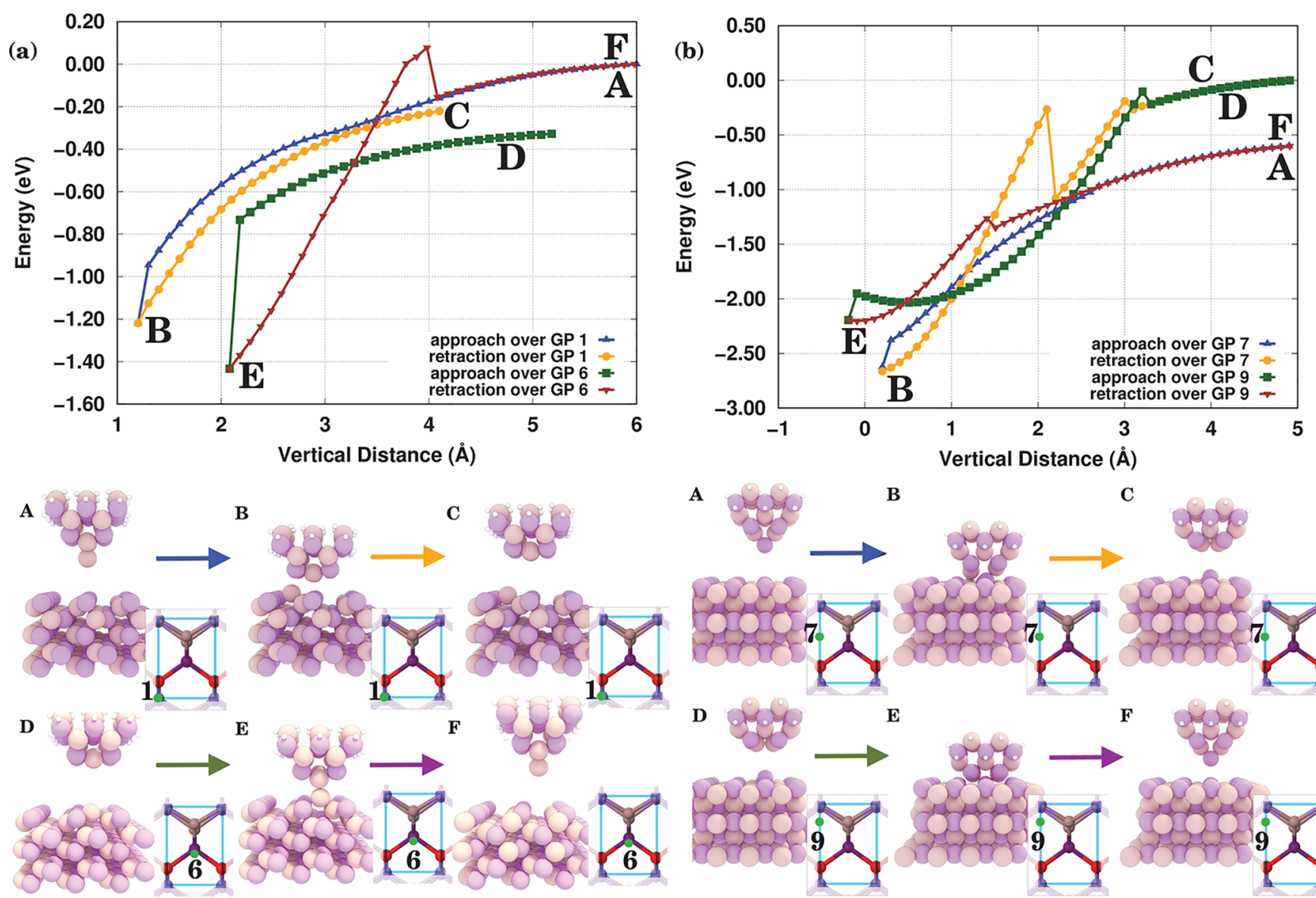

A

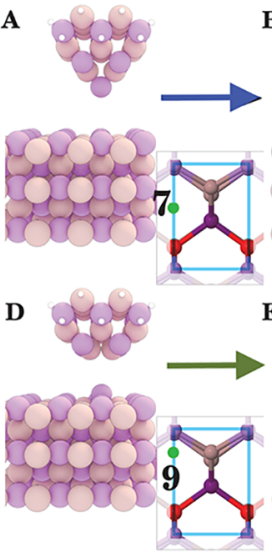

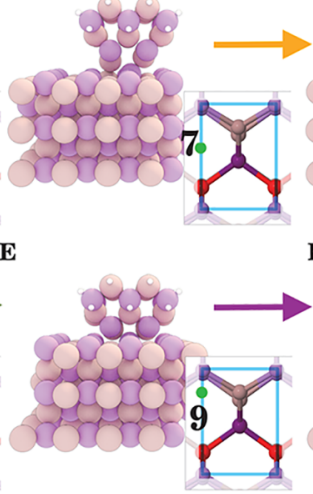

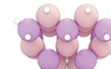

Figure 3. Energy curves (top panels) and the corresponding geometries (bottom panels) at the characteristic points of the full picking-deposition cycle for (a) gallium and (b) arsenic atoms. In both cases the same blunt tip was used; that is, the tips in (a, b) only differ by their apex atom.

We shall first focus on the four-gold-atom-doped GaAs tip 4 of Figure 1d. At the tip apex we have an $\mathrm{Au}$ atom. Our calculations show that this atom can be deposited on the surface within a window of heights of $\Delta z=2-3 \AA$ above grid points (GPs) 4, 5, and 7. Once the tip has deposited its apex atom, it becomes blunt with only three gold atoms left that are exposed to the surface (Figure 1d, tip 5). As explained below, we find that this blunt tip is capable of collecting another gold adatom from the GaAs surface, and in doing so it recovers its original structure, ready to deposit again the acquired gold atom, thereby performing a complete pick-deposit cycle. We demonstrate this by placing a gold adatom on the surface and then performing with the blunt tip approach-retraction cycles over each of the ten grid points (GPs). After performing retraction from different heights $z$, we find that for some of them (specific window of turning points heights $z$ ) the gold adatom can be collected by the blunt tip when placed above GPs 9 and 10. In doing so the original tip structure of Figure $1 \mathrm{~d}, 4$ is fully restored.

The energy curves for all of these processes, together with characteristic geometries, are shown in Figure 2a. The hybrid tip is approached over GP 7 (the blue curve), and a sudden change in the energy curve appears at around $z \simeq 2.7 \AA$ due to a strong interaction between the gold apex atom and the surface, as shown in geometry B in Figure 2a. After retraction of the tip (the yellow curve), the gold atom remains on the surface ( $\mathrm{C}$ in Figure 2a) and the tip becomes blunt. Next, we move the blunt tip from GP 7 to GP 10 to perform a new approach-retraction cycle (sequence $\mathrm{D} \rightarrow \mathrm{E} \rightarrow \mathrm{F}$ ). This time, at a lower height, two sudden changes are observed, indicating that the collection mechanism is not a single-step process (the green curve). The adatom is attached to the tip at $z \simeq 2.1$ ( $\mathrm{E}$ in Figure 2a), and after retraction (the red curve) the $\mathrm{Au}$ atom stays on the tip, completing the full pick-deposit cycle. As can be seen (compare geometries A and F), the tip structure has been completely restored.

The implication of this in silico experiment is that a class of GaAs-based tips exists, with a few $\mathrm{Au}$ atoms at their apexes, that can be transformed into a blunt tip by depositing a protruding gold atom. This blunt tip is capable of picking up a gold atom anywhere from the surface and then depositing it back at another location, restoring the blunt tip. This procedure can be performed, at least in theory, an arbitrary number of times, providing a proof of principle for rearranging gold atoms on the GaAs surface, one by one, with a single tip. Each elementary stage of the cycle can be performed with full control since we know precisely where on the surface the tip should approach the deposited atom to pick it up successfully and where it should approach the surface to deposit it back.

What makes this tip even more interesting is that it can deposit a gold atom next to another one on the surface forming a surface dimer, as shown in Figure $2 \mathrm{~b}$. This in silico experiment has particular potential in guiding and informing the experimental atom-by-atom fabrication of a $2 \mathrm{D}$ cluster of gold atoms on the GaAs surface. 
The hybrid GaAsAu tip can, however, perform a rather limited function as it is capable of completing the full pickingdeposition cycle only with gold atoms. A class of other tips exists, however, that can perform the full picking-deposition cycle with more than one species. This is essential if we want to eventually build nanostructures made of various species, as in this case we would need to be able to manipulate different individual atoms in a repetitive manner and with the same tip. We find that at least one blunt GaAs tip exists (tip 3 in Figure 1d) that is capable of reversibly picking up an atom from the surface into its apex position and then placing it back somewhere on the surface, regardless of the chemical nature of the atom.

The important details associated with these manipulation processes are shown in Figure 3. In the left (right) panel, manipulation of a Ga (As) atom is shown. Each state of the system during the manipulation cycle, when the tip is at either the farthest or the closest point to the surface, is designated by letters from $A$ to $F$. In the top panels the energy-distance curves are given, while below these we show the geometries for the states from A to F.

As before, it is convenient to start from the atomically sharp tip 1, which is composed of the blunt tip 3 (Figure 1d), with the $\mathrm{Ga}$ atom at its apex. Initially, this tip is positioned above GP 1 (see point A in Figure 3a). On pushing the tip into the surface (transition $A \rightarrow B$ ) (the blue curve), the apex Ga atom jumps from the tip to the surface (state B). After retraction of the tip (the yellow curve), the adatom remains on the surface, and the tip, now being blunt, still keeps the basic structure of Figure 1d,3 (state C). Interestingly, the Ga atom during this manipulation settles initially in a metastable position on the surface that is by $0.2 \mathrm{eV}$ less stable than the global minimum structure. However, because of a very small energy barrier of $17 \mathrm{meV}$ (see details in the Supporting Information), even at rather low temperatures, we should expect the adatom to jump rather quickly into the most stable position on the surface. Hence, we find that this procedure leads to $\mathrm{Ga}$ atom deposition at the most stable lattice site via a two-step process.

Surprisingly, upon a more careful investigation, we find that, in addition to the deposition path considered above, there is another pathway that does not lead to an atom being deposited. The former path is slightly more favorable at large heights $(z \geqslant 3 \AA)$ at the onset of interaction with the surface, but as the tip gets closer to the surface $(z \leqslant 2 \AA)$, the second path becomes more favorable (see the Supporting Information). The calculated energy barrier connecting the first path with the second, calculated at $z=2 \AA$, was found to be $0.1 \mathrm{eV}$, which will be significant at low temperatures. Hence, if appropriate experimental conditions are maintained (sufficiently low temperature), the tip relaxation during the approach will follow the former (metastable) path, eventually leading to $\mathrm{Ga}$ atom deposition.

We subsequently moved the blunt tip to a different lateral position (above GP 6 on the surface) and then approached almost $2.0 \AA$ toward the surface ( $\mathrm{D} \rightarrow \mathrm{E}$, the green curve). One can see that at this close approach the Ga adatom, placed in the most stable geometry on the surface, jumps back to the tip (E). Next, the tip was retracted (red curve) and the original Ga-terminated tip structure fully recovered (state F, with the total energy practically identical with that of A). Hence, similar to the GaAsAu hybrid tip, the full vertical single atom manipulation cycle has been performed: deposition above one grid point and picking up at another. It is important that the structures of the tip at the beginning and the end of the cycle are identical, meaning that this cycle can be performed any number of times, repetitively. Moreover, similar to the GaAsAu tip, the deposition of the $\mathrm{Ga}$ atom can be considered as a method of formation of a blunt reactive tip, with subsequent retraction-deposition cycles serving to pick up $\mathrm{Ga}$ atoms anywhere on the surface and deposit them elsewhere.

Taking now the tip of Figure 1d,2, which contains an As atom at its apex instead, a very similar-in-spirit manipulation cycle with this tip is shown in Figure $3 \mathrm{~b}$. Initially the tip is prepared above GP 7 and then driven into the surface $(\mathrm{A} \rightarrow$ B), depositing its apex As atom directly in the most stable position (blue curve for approach and yellow $\mathrm{B} \rightarrow \mathrm{C}$ for retraction). Note that there is an abrupt change in the energy seen in the retraction curve corresponding to the atom jumping to the surface at $z \simeq 2.2 \AA$. Next, the tip is moved laterally above GP 9 (D), and then after the approach $(\mathrm{D} \rightarrow \mathrm{E}$, the green curve) and retraction $(\mathrm{E} \rightarrow \mathrm{F}$, the red curve) the cycle is repeated, during which the dropped As atom is collected back onto the tip and the latter returns to its original structure.

It is essential that in both cases of the Ga- and As-terminated tips the structure of the blunt tip, which serves as the common base to both tips, after the apex atom is deposited onto the surface (states C and D in both cases), is exactly the same. Hence, once the blunt tip is generated by depositing its apex atom (that is, being either Ga or As), this tip is capable of manipulating vertically either of the two species, repeatedly and in any order, on the GaAs(110) surface.

Although both Ga and As atoms can be manipulated by using exactly the same blunt tip, they are not manipulated in exactly the same way. A closer look at the energy curves of Figure 3 reveals some differences. First, as was stated above, Ga and As atoms have to be deposited over different grid points (1 for $\mathrm{Ga}$ and 7 for As). Second, for the Ga-apex tip the deposition happens around $z=1.2 \AA$, while for the Asterminated tip the jump in energy due to deposition happens at an even smaller tip-sample separation, $z=0.2 \AA$. The same situation happens for the removal process, which for Ga and As happens over grid points 6 and 9, respectively. Third, Ga deposition happens at lower $z$ than in the removal process, while in the As case this situation is inverted. Finally, Ga is deposited into a metastable state, while the As atom bonds in the most stable state.

Intriguingly, if either of the sharp tips is brought even closer to the surface (not shown), atom deposition does not happen. For example, for As deposition over GP 7 there is a window of distances, $\Delta z$, where deposition occurs. If the apex is not close enough to the surface, the apex atom remains on the tip; if the tip is too close to the surface, the atom also remains on the tip upon retraction. We speculate that this is due to the repulsion between the $\mathrm{Ga}$ atoms at the apex of the blunt tip and those on the surface: inside that $z$ window, the Ga atoms of the surface attract the As apex atom sufficiently strongly to retain it on the surface. If, however, the tip is moved closer toward the surface, the repulsion between the Ga atoms of the surface and of the tip dominates, and as a result, after retraction the As apex atom remains on the tip.

Furthermore, we have explored the possibility of manipulating five more species- $\mathrm{Al}$, In, $\mathrm{Sb}, \mathrm{P}$, and $\mathrm{Au}$-with the same blunt GaAs tip. For each of them, we have put the corresponding atom at the apex of the tip of Figure $1 d, 3$ and repeated the same exploratory computational protocol as 


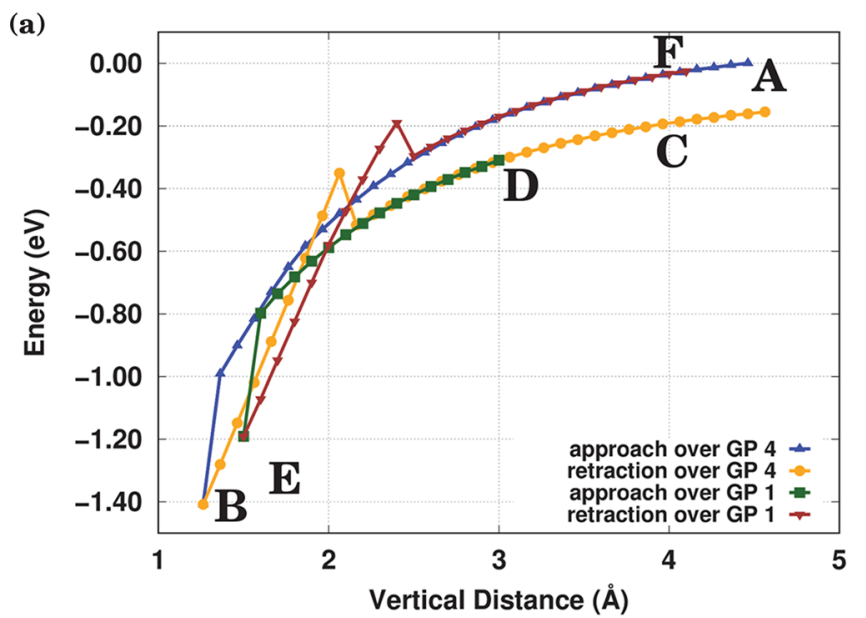

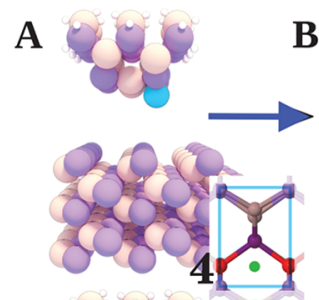

B
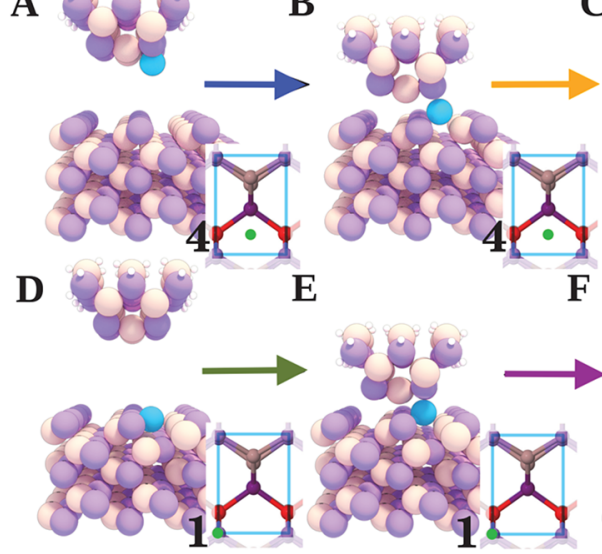

$\mathbf{E}$

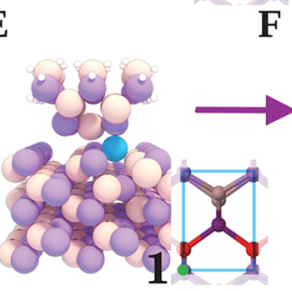

C
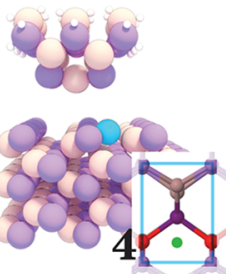

$\mathrm{F}$

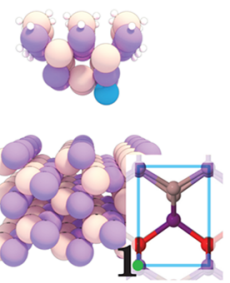

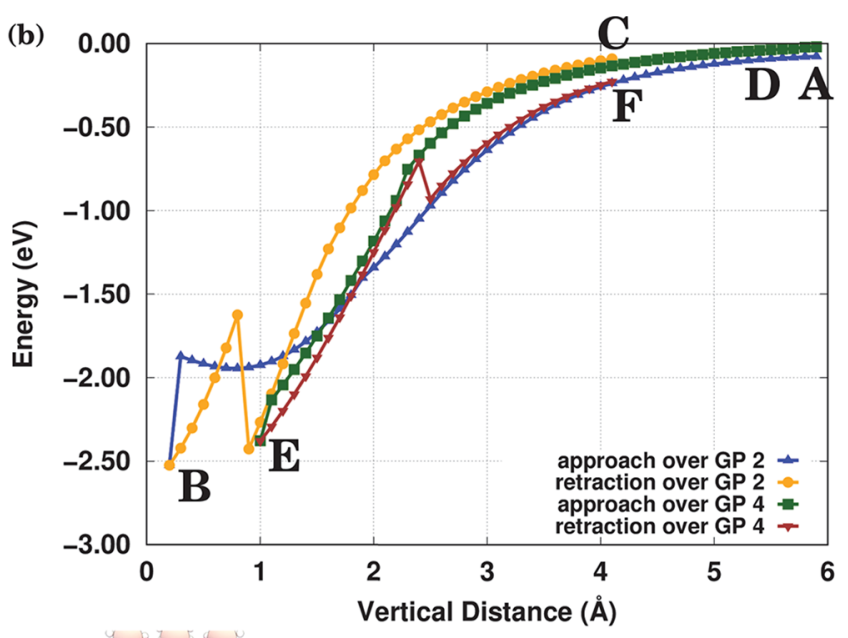

A

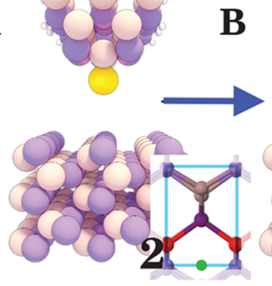

D

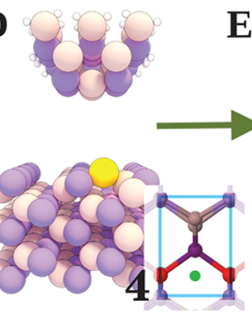

C
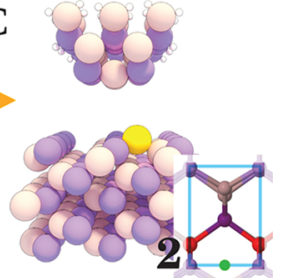

$\mathbf{F}$

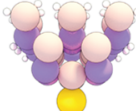

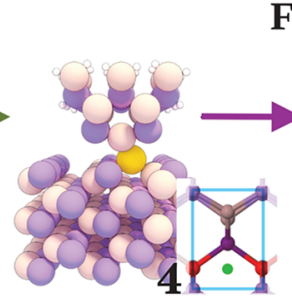

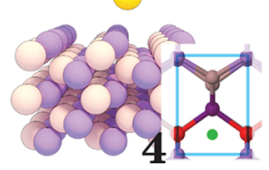

Figure 4. Energy curves for the full picking-deposition cycle for (a) Al (blue) and (b) Au (yellow).

before. Approaching and retracting the tip from different heights at different lateral positions shows that it is also possible to deposit $\mathrm{Al}$ and $\mathrm{Au}$. For $\mathrm{Al}$ (Figure 4a), we observed that the atom is adsorbed at the side of the tip. We then performed the approach-retraction protocol to find that the apex atom can also be deposited at the most stable adsorption site by approaching the tip over GP 4, and an atom can be picked up over GP 1 . That means that a full manipulation cycle with the same blunt tip can also be performed for Al. We have also successfully manipulated a Au atom with the same tip, as shown in Figure 4b: the Au atom can be deposited over GP 2 $(\mathrm{A} \rightarrow \mathrm{B} \rightarrow \mathrm{C})$ and collected from GP $4(\mathrm{D} \rightarrow \mathrm{E} \rightarrow \mathrm{F})$.

The vertical manipulation of $\mathrm{In}, \mathrm{Sb}$, and $\mathrm{P}$ with our GaAs blunt tip has been found unsuccessful (see the Supporting Information for details).

The core result of this paper is that there is a class of AFM tips-such as the ones with a blunt stoichiometric GaAs apex-that can manipulate not only atoms of its own species but also other atoms, such as $\mathrm{Au}$ and $\mathrm{Al}$ in a repetitive manner. The implications of this are profound. It means that the same (blunt) tip can be used for picking up and depositing either of the four species, in any order and repeatedly, opening a wayat least in theory-for building nanostructures with these atoms on the GaAs(110) surface with atomic precision within the same experimental run. Similarly, a Au-doped GaAs tip was found that can deposit and pick up at least a gold atom, also repeatedly, suggesting that there exists a class of such tips capable of repeated vertical manipulation. It is essential to note that manipulation of single atoms with the same tip relies on a very detailed balance of physical interactions that is especially sensitive to the particular lateral and vertical tip positions and tip model.

Our computational protocol, or a similar one, can be employed in future calculations of finding wider families of possible tips capable of described picking-deposition cycles. However, the important outcome of this work is that our conclusions pave the way to establish experimental guidelines for cyclical vertical single atom manipulations of different species on a nonmetallic surface. More explicitly, the following directives can be drawn from our work:

(i) A cyclic manipulation requires a blunt reactive tip. An example of such a tip is a GaAs cluster shown in Figure 1d,3. To fabricate such a tip, first an indentation of the tip into the surface is required until stable atomic resolution is achieved. Then, the required blunt tips can be prepared by removing apex atom(s), thereby turning them into blunt tips with enhanced reactivity. Similarly, the preparation of tip apexes with species different from those of the surface itself (as, for instance, gold-terminated tips of Figure 1d,4) require indenting the tip into surface clusters made of the desired species.

(ii) The tips must be reactive in a sense that the interaction of a surface adatom is stronger with the tip than with the surface at some lattice sites (so that this atom can be picked up by the tip) and weaker at the others (so it can be dropped). Theoretical simulations are necessary to guide the experimentalists in selecting the appropriate lattice positions for picking 
and depositing adatoms. Examples of "successful" tips are provided by the blunt GaAs and the hybrid GaAsAu tips discussed here. A detailed analysis of breaking and formation of bonds could shed some light over the chemistry involved in the processes.

(iii) Because of the critical dependence of the atomic manipulation probability on the position of the tip within the surface unit cell, a 2D grid of frequency shift measurements is first required. Because of the precision required to locate the various grid points within the unit cell, drift compensation will almost certainly be necessary.

(iv) There is a certain window of tip heights $\Delta z$ at which either of the manipulations happens.

(v) Finally, the stiffness of the tip should be comparable to that of the surface: a softer tip (e.g., a pure gold tip) would not be able to pick up atoms from the GaAs surface (see, however, the swapping mechanism considered in the Supporting Information), while a stiffer tip (e.g., a tungsten tip; see the Supporting Information) would disrupt the surface extensively, extracting atoms in an uncontrollable fashion with little hope of redeposition. Therefore, tips made of the same material as the surface, or similar, are advantageous.

The manipulation protocols require precise positioning of the tip with respect to the manipulated species (pick up) or the lattice site (deposition). In turn, this requires, at all stages of the manipulation process, that the surface can be imaged with high resolution. However, as the apex atom is lost, the tip becomes blunt and will lose this ability. This reduction in image resolution will follow an abrupt change in energy (Figure 3). Hence, the key signature of deposition of an apex atom and the primary "observable" for experimentalists is the abrupt change in the force-distance curve and the concomitant reduction in image resolution that will accompany this change. Because of its smaller radius of curvature, the deposited atom will act to "reverse image" the blunter tip. This will make the interpretation of the image contrast somewhat problematic, but nonetheless, a very distinct feature related to the deposited atom will be observed.

Because of the requirement of the precise positioning of the tip with respect to the surface, one has to rely on low temperatures or an atom-tracking system to avoid thermal drift and an ability to program all stages of the manipulation process after taking an image of the manipulating area with a sharp tip at the start of the manipulation sequence; $77 \mathrm{~K}$ is feasible, but $4 \mathrm{~K}$ is preferable. Low temperatures may also be essential to fix the desired manipulation path (as is the case with Ga atom deposition). Also, it is known ${ }^{34}$ that adsorbates are very easily translated laterally across the surface by the tip unless the temperatures are sufficiently low.

The key proof of principle of the cyclic manipulation process will be the readsorption of the deposited atom on the blunt tip. For $\mathrm{Ga}$, this produces a striking discontinuity in the $E(z)$ curve (compare curve $\mathrm{D} \rightarrow \mathrm{E}$ in Figure 3a) associated with a change in energy of $0.75 \mathrm{eV}$ when the atom returns to the tip. Accompanying this discontinuity will be a restoration of highresolution imaging.

The identification of tips that are indefinitely "recyclable" is an exciting new addition to the atomic manipulation toolbox. Our simulations have predicted characteristic energy vs distance signatures for this type of tips, and we are hopeful that those predictions will inspire experimentalists to verify them in the near future.

\section{ASSOCIATED CONTENT}

\section{Supporting Information}

The Supporting Information is available free of charge at https://pubs.acs.org/doi/10.1021/acs.jpclett.1c02271.

Extracting atoms from the upper $\mathrm{GaAs}(110)$ surface layer; adsorption energies and geometries of $\mathrm{Ga}$ and As adatoms on the GaAs(110) surface; selection of extra tips; alternative GaAs tip approaching path; Ga adatom diffusion energy barrier; deposition with pure gold tips: (i) swapping between Au tip and As surface atoms, (ii) gold thread formation on the GaAs surface; approaching-retraction cycle of GaAs tips with In, $\mathrm{P}$, and $\mathrm{Sb}$ at the apex; approaching-retraction cycle of a pure $\mathrm{W}$ tip; computational methods (PDF)

\section{AUTHOR INFORMATION}

\section{Corresponding Authors}

David Abbasi-Pérez - Department of Physics, King's College London, London WC2R 2LS, U.K.; ㅇ orcid.org/00000003-1035-9458; Email: david.abbasi_perez@kcl.ac.uk

Lev Kantorovich - Department of Physics, King's College London, London WC2R 2LS, U.K.; (1) orcid.org/00000001-9379-6834; Email: lev.kantorovitch@kcl.ac.uk

\section{Authors}

Hongqian Sang - Department of Physics, King's College London, London WC2R 2LS, U.K.; Institute for Interdisciplinary Research, Jianghan University, Wuhan 430056, China; 이이.org/0000-0003-2738-8861

Filipe L. Q. Junqueira - School of Physics and Astronomy, University of Nottingham, Nottingham NG7 2RD, U.K.

Adam Sweetman - School of Physics \& Astronomy, University of Leeds, Leeds LS2 9JT, U.K.

J. Manuel Recio - MALTA-Consolider Team and Department of Analytical and Physical Chemistry, Universidad de Oviedo, Oviedo 33006, Spain; (1) orcid.org/ 0000-0002-3182-7508

Philip Moriarty - School of Physics and Astronomy, University of Nottingham, Nottingham NG7 2RD, U.K.; 10 orcid.org/ 0000-0002-9926-9004

Complete contact information is available at:

https://pubs.acs.org/10.1021/acs.jpclett.1c02271

\section{Notes}

The authors declare no competing financial interest.

\section{ACKNOWLEDGMENTS}

Via our membership of the UK's HEC Materials Chemistry Consortium, which is funded by EPSRC (EP/L000202, EP/ R029431), this work used both the ARCHER UK National Supercomputing Service (http://www.archer.ac.uk) and the UK Materials and Molecular Modelling Hub for computational resources, MMM Hub, which is partially funded by EPSRC (EP/P020194). This research was supported by the UK EPSRC Grant EP/N023587/1. H.S. acknowledges the support from the National Natural Science Foundation of China (Grant 21603086) and the China Scholarship Council (Grant 201608420186). J.M.R. thanks the Spanish MINECO and FICYT for financial support (Projects PGR2018-094814-BC22 and FC-GRUPIN-IDI/2018/000177) and Universidad de Oviedo for a mobility grant to visit King's College London. 


\section{REFERENCES}

(1) Binnig, G.; Rohrer, H.; Gerber, Ch.; Weibel, E. Tunneling through a controllable vacuum gap. Appl. Phys. Lett. 1982, 40, 178180.

(2) Binnig, G.; Rohrer, H.; Gerber, Ch.; Weibel, E. Surface Studies by Scanning Tunneling Microscopy. Phys. Rev. Lett. 1982, 49, 57-61.

(3) Binnig, G.; Quate, C. F.; Gerber, Ch. Atomic Force Microscope. Phys. Rev. Lett. 1986, 56, 930-933.

(4) Custance, O.; Perez, R.; Morita, S. Atomic force microscopy as a tool for atom manipulation. Nat. Nanotechnol. 2009, 4, 803.

(5) Eigler, D. M.; Schweizer, E. K. Positioning single atoms with a scanning tunnelling microscope. Nature 1990, 344, 524-526.

(6) Whitman, L. J.; Stroscio, J. A.; Dragoset, R. A.; Celotta, R. J. Manipulation of Adsorbed Atoms and Creation of New Structures on Room-Temperature Surfaces with a Scanning Tunneling Microscope. Science 1991, 251, 1206-1210.

(7) Avouris, Ph.; Lyo, I.-W. Probing and inducing surface chemistry with the STM: the reactions of $\mathrm{Si}(111)-7 \times 7$ with $\mathrm{H}_{2} \mathrm{O}$ and O2. Surf. Sci. 1991, 242, 1-11.

(8) Avouris, Ph.; Walkup, R. E.; Rossi, A. R.; Akpati, H. C.; Nordlander, P.; Shen, T.-C.; Abeln, G. C.; Lyding, J. W. Breaking individual chemical bonds via STM-induced excitations. Surf. Sci. 1996, 363, 368-377.

(9) Jung, T. A.; Schlittler, R. R.; Gimzewski, J. K. Conformational identification of individual adsorbed molecules with the STM. Nature 1997, 386, 696-698.

(10) Martsinovich, N.; Hobbs, C.; Kantorovich, L.; Fawcett, R. H. J.; Humphry, M. J.; Keeling, D. L.; Beton, P. H. Manipulation of $\mathrm{C}_{60}$ on the $\mathrm{Si}(001)$ surface: Experiment and theory. Phys. Rev. B: Condens. Matter Mater. Phys. 2006, 74, 085304.

(11) Ternes, M.; Lutz, C. P.; Hirjibehedin, C. F.; Giessibl, F. J.; Heinrich, A. J. The Force Needed to Move an Atom on a Surface. Science 2008, 319, 1066-1069.

(12) Huff, T. R.; Labidi, H.; Rashidi, M.; Koleini, M.; Achal, R.; Salomons, M. H.; Wolkow, R. A. Atomic White-Out: Enabling Atomic Circuitry through Mechanically Induced Bonding of Single Hydrogen Atoms to a Silicon Surface. ACS Nano 2017, 11, 8636-8642.

(13) Oyabu, N.; Custance, Ó.; Yi, I.; Sugawara, Y.; Morita, S. Mechanical Vertical Manipulation of Selected Single Atoms by Soft Nanoindentation Using Near Contact Atomic Force Microscopy. Phys. Rev. Lett. 2003, 90, 176102.

(14) Oyabu, N.; Sugimoto, Y.; Abe, M.; Custance, Ó.; Morita, S. Lateral manipulation of single atoms at semiconductor surfaces using atomic force microscopy. Nanotechnology 2005, 16, S112-S117.

(15) Sugimoto, Y.; Pou, P.; Abe, M.; Jelinek, P.; Pérez, R.; Morita, S.; Custance, Ó. Chemical identification of individual surface atoms by atomic force microscopy. Nature 2007, 446, 64.

(16) Giessibl, F. J. High-speed force sensor for force microscopy and profilometry utilizing a quartz tuning fork. Appl. Phys. Lett. 1998, 73, 3956-3958.

(17) Giessibl, F. J.; Bielefeldt, H.; Hembacher, S.; Mannhart, J. Calculation of the optimal imaging parameters for frequency modulation atomic force microscopy. Appl. Surf. Sci. 1999, 140, 352-357.

(18) Giessibl, F. J.; Hembacher, S.; Herz, M.; Schiller, Ch; Mannhart, J. Stability considerations and implementation of cantilevers allowing dynamic force microscopy with optimal resolution: the qPlus sensor. Nanotechnology 2004, 15, S79-S86.

(19) Giessibl, F. J. The qPlus sensor, a powerful core for the atomic force microscope. Rev. Sci. Instrum. 2019, 90, 011101.

(20) Sweetman, A.; Jarvis, S.; Danza, R.; Bamidele, J.; Gangopadhyay, S.; Shaw, G. A.; Kantorovich, L.; Moriarty, P. Toggling Bistable Atoms via Mechanical Switching of Bond Angle. Phys. Rev. Lett. 2011, 106, 136101.

(21) Pavlicek, N.; Schuler, B.; Collazos, S.; Moll, N.; Pérez, D.; Guitián, E.; Meyer, G.; Peña, D.; Gross, L. On-surface generation and imaging of arynes by atomic force microscopy. Nat. Chem. 2015, 7, 623.
(22) Pavlicek, N.; Majzik, Z.; Collazos, S.; Meyer, G.; Pérez, D.; Guitián, E.; Peña, D.; Gross, L. Generation and Characterization of a meta-Aryne on $\mathrm{Cu}$ and $\mathrm{NaCl}$ Surfaces. ACS Nano 2017, 11, 1076810773.

(23) Gross, L.; Mohn, F.; Moll, N.; Liljeroth, P.; Meyer, G. The Chemical Structure of a Molecule Resolved by Atomic Force Microscopy. Science 2009, 325, 1110-1114.

(24) Bamidele, J.; Li, Y. J.; Jarvis, S.; Naitoh, Y.; Sugawara, Y.; Kantorovich, L. Complex design of dissipation signals in non-contact atomic force microscopy. Phys. Chem. Chem. Phys. 2012, 14, 1625016257.

(25) Sweetman, A. M.; Jarvis, S. P.; Sang, H.; Lekkas, I.; Rahe, P.; Wang, Yu; Wang, J.; Champness, N. R.; Kantorovich, L.; Moriarty, P. Mapping the force field of a hydrogen-bonded assembly. Nat. Commun. 2014, 5, 3931.

(26) Bamidele, J.; Lee, S. H.; Kinoshita, Y.; Turansky, R.; Naitoh, Y.; Li, Y. J.; Sugawara, Y.; Stich, I.; Kantorovich, L. Vertical atomic manipulation with dynamic atomic-force microscopy without tip change via a multi-step mechanism. Nat. Commun. 2014, 5, 4476.

(27) Rashidi, M.; Wolkow, R. A. Autonomous Scanning Probe Microscopy in Situ Tip Conditioning through Machine Learning. ACS Nano 2018, 12, 5185-5189.

(28) Gordon, O.; D’Hondt, P.; Knijff, L.; Freeney, S. E.; Junqueira, F.; Moriarty, P.; Swart, I. Scanning tunneling state recognition with multi-class neural network ensembles. Rev. Sci. Instrum. 2019, 90, 103704.

(29) Gordon, O. M; Junqueira, F. L Q; Moriarty, P. J Embedding human heuristics in machine-learning-enabled probe microscopy. Mach. Learn.: Sci. Technol. 2020, 1, 015001.

(30) Jarvis, S.; Sweetman, A.; Bamidele, J.; Kantorovich, L.; Moriarty, P. Role of orbital overlap in atomic manipulation. Phys. Rev. B: Condens. Matter Mater. Phys. 2012, 85, 235305.

(31) Sugimoto, Y.; Pou, P.; Custance, O.; Jelinek, P.; Abe, M.; Perez, R.; Morita, S. Complex Patterning by Vertical Interchange Atom Manipulation Using Atomic Force Microscopy. Science 2008, 322, 413-417.

(32) Kawai, S.; Foster, A. S.; Canova, F. F.; Onodera, H.; Kitamura, S.; Meyer, E. Atom manipulation on an insulating surface at room temperature. Nat. Commun. 2014, 5, 4403.

(33) Kinoshita, Y.; Turanský, R.; Brndiar, J.; Naitoh, Y.; Li, Y. J.; Kantorovich, L.; Sugawara, Y.; Stich, I. Promoting atoms into delocalized long-living magnetically modified state using atomic force microscopy. Nano Lett. 2016, 16, 7490-7494.

(34) Kaku, S.; Yoshino, J. Temperature-Dependent Tip-Induced Motion of Ga Adatom on GaAs(110) Surface. Small 2020, 16, 2002296. 